\title{
Preparing Accelerator Systems for the RHIC Sextant Commissioning *
}

\author{
D. Trbojevic, F. Pilat, L.Ahrens, D.Barton, T. Clifford, R.Connoly, W. Fischer, M. Harrison, \\ W. MacKay, R. Olsen, S. Peggs, T. Satogata, S. Tepikian, P. Thompson, C.G. Trahern, and R. Witkover \\ Brookhaven National Laboratory, Upton, NY, 11973, USA
}

\section{Abstract}

The Relativistic Heavy Ion Collider (RHIC) construction is progressing steadily towards completion in 1999 when beams will circulate in both collider rings. One of the major tests of the RHIC project was the commissioning of the first sextant with gold ion beams in early 1997 . This is a report on preparation of the RHIC accelerator systems for the first sextant test. It includes beam position monitors, timing, injection correction through the magnetic septum and kickers, current transformers, "flags" and the ionization beam profile monitors, beam loss monitors, beam and quench permit link system, power supply controls, and the configuration database system. The software and hardware development and coordination of the different systems before commissioning were regularly checked during bi-weekly, and (later) weekly, progress report meetings.

\section{INTRODUCTION}

The Relativistic Heavy Ion Collider (RHIC) construction has reached its final stage. The sextant test represents a crucial proof of principle for the whole RHIC machine. This report describes preparations of the accelerator systems for the test. It includes: Beam profile monitors (flags), Loss monitors (BLM), Beam current transformers (BCM), Beam and quench permit links, Power supply configurations; Ionization profile monitor (IPM), Injection kickers, Timeline events, Beam position monitors (BPM), and Database configuration. A goal of the RHIC sextant test was to verify the machine design and system performance by transporting a heavy-ion gold beam from the Alternating Gradient Synchrotron (AGS) through the already commissioned $U$ and $W$ lines - ATR, through the new Y-arc line, the magnetic septum-Lambertson magnet, through the sextant arc of the yellow ring, pass through the operating RF $200 \mathrm{MHz}$ cavity, and through the high focusing triplet and $D O$ dipole magnets to reach a temporary beam dump. The sextant test required the same operating conditions as at RHIC injection (every magnet is at $4 \mathrm{~K}$ temperature). During the test only the yellow ring was connected to the power supplies. The accelerator systems had to be prepared to steer the gold beam, to be able to measure the phase advance per cell, beam profiles, vertical and horizontal transverse emittances, transmission, losses etc. Preparations of the other systems in the sextant test are not mentioned in detail in this report. The test would not have been successful if magnets were not correctly installed, positioned, and electrically connected in the tunnel. This applies also to the

- Work performed under the auspices of the U.S. Department of Energy cryogenic, vacuum, control system, power supply systems, etc. Very complicated installation procedures of cryogenic lines, valve boxes, vacuum beam pipes and other insulating vacuum connections were all part of the preparation for the test. Separate reports are available describing surveying and alignment procedures in RHIC [1]. Details of the cryogenic system [2], magnet installation, vacuum system, and other systems are also reported elsewhere [3].

\section{SYSTEM PREPARATION}

Beam system commissioners were assigned before the sextant test and insured each system was progressing according to a coordinated plan. During preparation for the sextant test, sixteen commissioning meetings were held. This allowed integration between different systems and low and high level controls. At each meeting, first biweekly and later weekly, commissioners provided an update on their system with a separate report on a specific system.

- Beam Profile Monitors.

The beam profile monitors used in the test were the same type as during the AGS to RHIC beam line commissioning during the end of the 1995 (as previously reported [4]). Two additional profile monitors were installed at the end

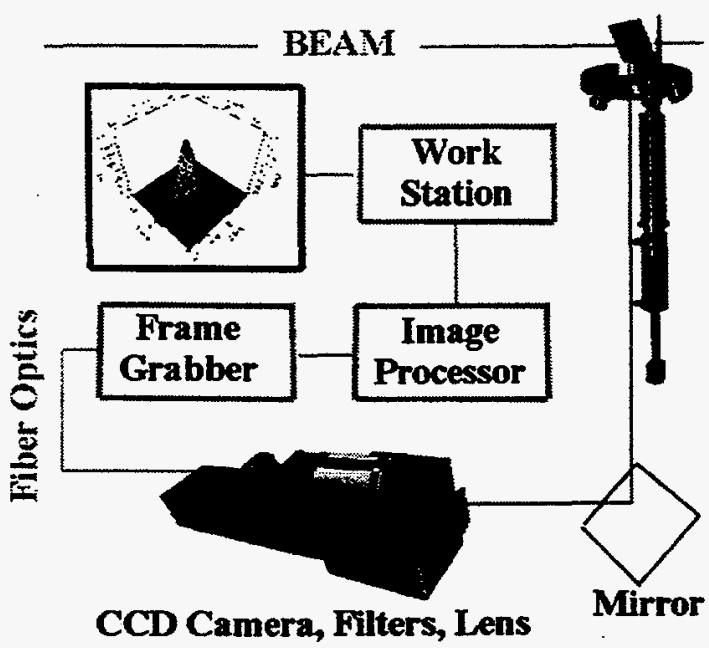

Figure 1: Beam Profile Monitor System

of the $y$-arc before the magnetic septum and at a temporary one at the end of the sextant. Preparation for the beam profile monitor system included new high level application software development which provided either two dimensional or even three dimensional beam profiles with inten- 
sity of the beam presented in the vertical axis. An automatic procedure fitted beam profiles to a Gaussian distribution, adjusting the $\sigma_{x}$ and $\sigma_{y}$ of the beams, the rotational angles of the beam ellipses in both $x-x^{\prime}$ and $y-y^{\prime}$ phase spaces, and the center of the beam position.(see the report at these proceedings [3]). The beam profile monitor system consists of a movable phosphor screen with mirror, CCD camera with filters, frame-grabber, image processor, and work station as shown in figure 1 . The beam loss monitor system (BLM) preparation for the sextant test included loss monitor bench calibration, installation in the RHIC tunnel, and signal checking. The sextant test operation of the system was in a single pass mode with many msec between the beam pulses, while for RHIC operations [5] different modes are being developed. The loss monitors were installed at each RHIC quadrupole. Every monitor was positioned between the two quadrupoles of parallel blue and yellow rings to record losses from both rings in the future.

\section{- Loss monitors}

Around the magnetic septum the Lambertson magnet and the injection kickers, more monitors were added. Signals from the loss monitors in the sextant test (more details are available in reference [5]) were brought to three service buildings where the integrator channels, high voltage power supplies, and MADC's (Multiplex Analog to Digital Converters) are located. Before the sextant test each monitor was tested in two ways from the control room. After the electrical connection was confirmed by a large resistor and applied voltage the signals from the MADC's were plotted by applying high voltage for a short time. The whole preparation procedure contributed to reliable system operation in the sextant test run [3].

\section{- Beam current transformers}

The beam current transformers used in the sextant test were of Integrating Current Transformer (ICT) type developed by Unser [7] for LEP. This design incorporates two toroidal transformer cores and is particularly suited to measuring the charge in short beam bunches. By passively stretching the initial puise, the signal is converted downward in frequency prior to applying it to the transformer. This overcomes core losses at high frequencies, while preserving the relative charge and, in addition, allows the electronics to be located further from the detector. The charge transformers allowed precision measurements (to $1 \%$ ) over a wide range of beam intensities. During the sextant test preparation two additional current transformers were installed: one upstream of the magnet septum at the end of the $y$-arc, and the second at the end of the sextant in front of the high focusing triplet quadrupoles.

\section{- Beam and quench protection permit links}

A new beam and quench permit link system has been designed for RHIC and partially commissioned during the sextant test. Full RHIC beam and quench protection permit links will be established when both rings are completed. A performance report of the system is presented elsewhere [3]. The system is also described in detail in reference [8]. The beam and quench permit links allowed beam entry into the RHIC sextant when RHIC accelerator support systems statuses were present. RHIC accelerator support systems include beam position and loss monitors, vacuum, power supply system status, and personnel safety. These systems report their status to the permit system. If any permit input or permit system connection fails, the beam permit system master module generates blue and yellow ring beam dump commands and triggers an abort event transmission. The $1 \mathrm{MHz}$ carrier is created in a master module and the link is established throughout all other modules and back to the master unit (see figure 2). There are three separate links: two quench protection links and one beam permit link. When one of the quench protection links is broken the whole beam permit link goes down. In the sextant test the three links were established around the RHIC sextant arc with a master and eight slave permit modules installed in the service buildings. An application program was developed during the preparation which displays the status of the link, location of a failure if occured, and the time stamp when the failure occured. A failure detected by the permit link in the sextant induces the shut-down of power to the switching magnet at the end of the previously commissioned $w$-line. This stops beam injection to RHIC.

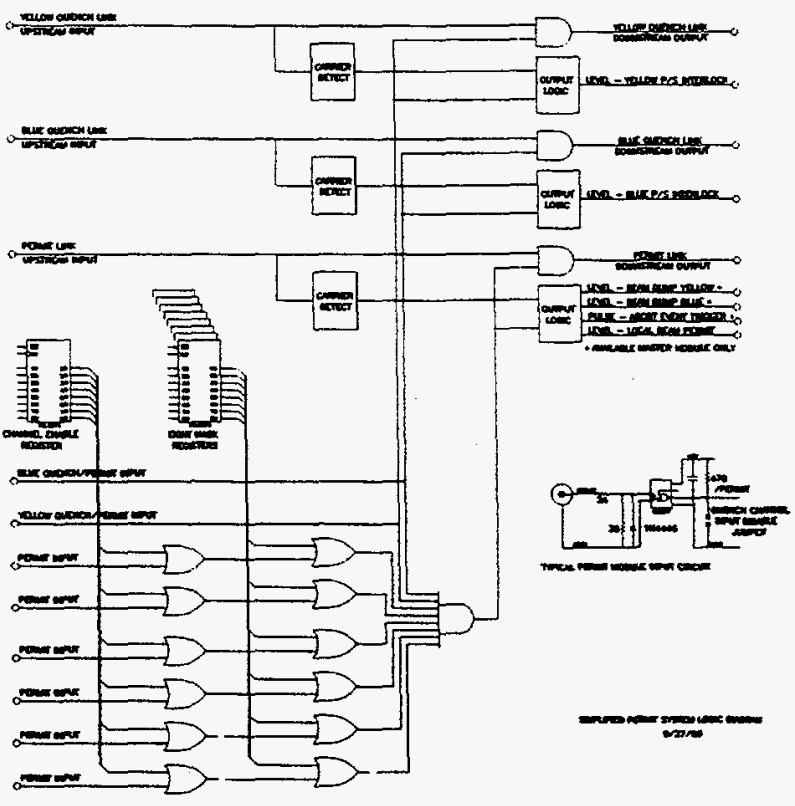

Figure 2: Beam and quench protection permit links logic diagram.

\section{- Power Supply configurations}

The ATR power supplies were provided to the: switching magnet, combined function magnets in the $y$-arc, the Lambertson magnet. Two separate quad buses, a dipole bus, 
and every horizontal and vertical correction magnet were provided for the sextant. There was an additional trim quad magnet close to the end of the sextant connected to a power supply. More details about the lay-out and performance of the power supplies are reported elsewhere [3].

- Ionization profile monitor

A separate report [6] provides more details about this unique profile monitor developed during the preparation for the sextant test. The ionization profile monitor prototype was built to provide beam profiles from the electron signals which occur from the gold ion beam ionization of the residual gas in the beam vacuum pipe. A dipole magnet was used to prevent electron dispersal.

- Injection kickers

The injection kickers were not necessary for the sextant test because there was not circulating beam present and the beam could be steered by the correction magnets. But the whole injection kicker system was prepared on time to be completely commissioned during the test. A high level application code was developed during the preparation period to allow automatic tuning through the magnetic septum and the kickers as well as matching of the beam line to the RHIC.

\section{- Timeline events}

New RHIC beam events for the sextant test were established in addition to two existing AGS extraction events. These are three events for the wave form generators, and four events for the beam permit system: beam abort event, permit reset event, quench link reset event, and time stamp event to record the failure of the permit system.

- Beam position monitors-(BPM)

The BPM system will be operating in multi-turn mode when the RHIC accelerators are completed. The sextant test used them already in [9] one pass mode during commissioning of the ATR. Every corrector-quadrupolesextupoles (CQS) assembly has a BPM's installed and optically and electrically checked [1]. The surveying data of the BPM's were used by a high level application code to correct for a small misalignment with respect to the quads (of the order of $\sigma=0.6 \mathrm{~mm}$ ). During the sextant preparation period a completely new high level application code was developed. During the sextant test not every BPM was connected to the control system.

\section{- Database configuration}

Beginning with the AtR test in 1995, configuration databases were developed to provide data for both controls applications and general information with use of the SYBASE relational database management system. For the 1996 sextant test these AtR data structures were both replicated and developed further as the sextant test configuration database. During the preparation for the sextant test all systems (BPM, BLM, Power Supply, flags, etc.) were developed with appropriate connections to the database.

\section{CONCLUSIONS}

A well organized system integration and preparation for the sextant test was essential for successful operation. Although there were few delays in development of some systems with respect to the coordinated plan, the total sextant test preparation has been very successful. This was shown by the superb performance [3] of almost every system during the test operation.

\section{ACKNOWLEDGEMENTS}

We would like to especially thank: the people from the magnet group for making superb quality magnets, measuring them with a highest possible precision, and defining their electrical and magnetical properties without a single error; the vacuum and installation group who made this test possible by an extreme effort; the surveying group which aligned the element to a high precision, where the beam went through the sextant without the use of any corrector magnet; and the cryogenic group for highly professional performance.

\section{REFERENCES}

[1] D. Trbojevic, A. Jain, S. Tepikian, R. Grandinetti, G. Ganetis, J. Wei, and F. Karl, "Alignment of the High Beta Magnets in the RHIC Interaction Region", these proceedings.

[2] M. Iarocci, et.al., "RHIC $24 \mathrm{~kW}$ refrigerator and distribution system, construction, testing, and initial operating experience", these proceedings.

[3] F. Pilat, "RHIC Sextant Test - Accelerator Systems and Performance", 7P.05, these proceedings.

[4] R.L. Witkover, "Design of the Beam Profile Monitor System for the RHIC injection line", Proceeding of the 1995 Particle Accelerator Conference and International Conference on High- Energy Accelerators, held May 1-5, 1995, Dallas, Texas, pp. 2589-2591.

[5] R.L. Witkover, "RHIC Loss monitors", these proceedings.

[6] R. Connolly, " A Prototype Ionization Profile Monitor for RHIC.", 5P.47, these proceedings.

[7] K. B. Unser, "Measuring Bunch Intensity, Beam Loss and Bunch Lifetime in LEP", Proc. IEEE Particle Accelerator, Conference EPAC 90, Nice, France, June 1991, pp.786-788.

[8] R. Conkling, "V120 RHIC Beam Permit System Module", RHIC-Internal Report, (available on world wide web as: www.hichome.bnl.gov:80/Hardware/permit/permit.htm).

[9] P.R. Cameron, M.C. Grau, M. Morvillo, T.J. Shea, R.E. Sikora, "RHIC Beam Position Monitor Characterization", Proceedings of the 1995 PAC and International Conference on High Energy Accelerators, held May 1-5, 1995, Dallas, Texas, pp. 2458-2501. 
DISCLAIMER

This report was prepared as an account of work sponsored by an agency of the United States Government. Neither the United States Gnvernment nor any agency thereof, nor any of their empleyees, makes any warranty, express or implied, or assumes any legal liability or responsibility for the accuracy, completeness, or usefulness of any information, apparatus, product, or process disclosed, or represents that its use would not infringe privately owned rights. Reference herein to any specific commercial product, process, or service by trade name, trademark, manufacturer, or otherwise does not necessarily constitute or imply its endorsement, recommendation, or favoring by the United States Government or any agency thereof. The views and opinions of authors expressed herein do not necessarily state or reflect those of the United States Government or any agency thereof. 


\section{DISCLAMIER}

Portions of this document may be illegible in electronic image products. Images are produced from the best available original docoment. 